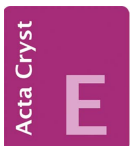

CRYSTALLOGRAPHIC COMMUNICATIONS

ISSN 2056-9890

Received 26 March 2020

Accepted 28 April 2020

Edited by W. T. A. Harrison, University of Aberdeen, Scotland

Keywords: crystal structure; 1,2,3-triazole; DFT calculation; Hirshfeld surface analysis.

CCDC reference: 1999643

Supporting information: this article has supporting information at journals.iucr.org/e
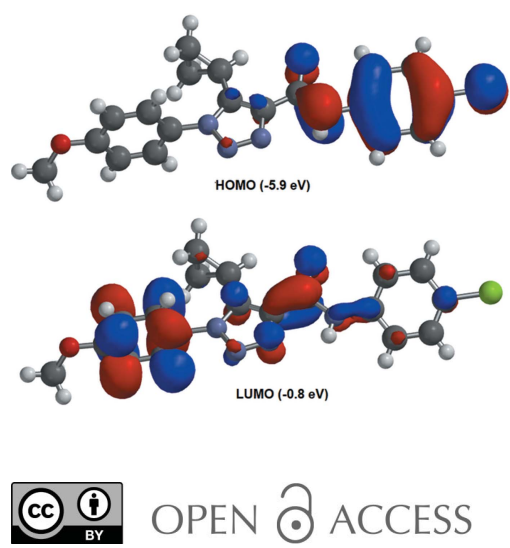

\section{Synthesis, crystal structure and Hirshfeld surface analysis of $\mathrm{N}$-(4-chlorophenyl)-5-cyclopropyl-1-(4- methoxyphenyl)-1H-1,2,3-triazole-4-carboxamide}

\author{
Nazariy Pokhodylo, ${ }^{\text {a* }}$ Yurii Slyvka ${ }^{\mathrm{b}}$ and Volodymyr Pavlyuk ${ }^{\mathrm{b}}$ \\ a Department of Organic Chemistry, Ivan Franko National University of Lviv, Kyryla i Mefodia Str, 6, 79005 L'viv, Ukraine, \\ and ${ }^{\mathbf{b}}$ Department of Inorganic Chemistry, Ivan Franko National University of Lviv, Kyryla i Mefodia Str, 6, 79005 L'viv, \\ Ukraine. *Correspondence e-mail: pokhodylo@gmail.com
}

The title compound, $\mathrm{C}_{19} \mathrm{H}_{17} \mathrm{ClN}_{4} \mathrm{O}_{2}$, was obtained via a two-step synthesis involving the enol-mediated click Dimroth reaction of 4-azidoanisole with methyl 3-cyclopropyl-3-oxopropanoate leading to the 5-cyclopropyl-1-(4-methoxyphenyl)-1H-1,2,3-triazole-4-carboxylic acid and subsequent acid amidation with 4-chloroaniline by $1,1^{\prime}$-carbonyldiimidazole (CDI). It crystallizes in space group $P 2_{1} / n$, with one molecule in the asymmetric unit. In the extended structure, two molecules arranged in a near coplanar fashion relative to the triazole ring planes are interconnected by $\mathrm{N}-\mathrm{H} \cdots \mathrm{N}$ and $\mathrm{C}-\mathrm{H} \cdots \mathrm{N}$ hydrogen bonds into a homodimer. The formation of dimers is a consequence of the above interaction and the edge-to-face stacking of aromatic rings, which are turned by $58.0(3)^{\circ}$ relative to each other. The dimers are linked by $\mathrm{C}-\mathrm{H} \cdots \mathrm{O}$ interactions into ribbons. DFT calculations demonstrate that the frontier molecular orbitals are well separated in energy and the HOMO is largely localized on the 4-chlorophenyl amide motif while the LUMO is associated with aryltriazole grouping. A Hirshfeld surface analysis was performed to further analyse the intermolecular interactions.

\section{Chemical context}

The number of compounds containing a 1,2,3-triazolyl-4carboxamide motif that are known to exhibit biological activity is increasing rapidly. At present, there are two approved drugs and a number of compounds are undergoing preclinical studies. For instance, rufinamide is a well-known drug among those currently marketed, which is used to treat Lennox-Gastaut syndrome (childhood-onset epilepsy) (Wheless \& Vazquez, 2010). Carboxyamidotriazole is a calcium channel blocker (Figg et al., 1995) and is currently being actively investigated as an anticancer drug in vitro (Bonnefond et al., 2018). As an example of preclinical anticancer studies, the cytotoxic activity at nanomolar levels of asymmetric $\quad 1-R-N-[(1-R-1 H-1,2,3$-triazol-4-yl)methyl $]-1 H-$ 1,2,3-triazole-4-carboxamides in B16 melanoma cells have been estimated (Elamari et al., 2013).

In our previous studies on the anticancer screening of various 1,2,3-triazoles, compounds based on 1,2,3-triazolyl-4carboxamide scaffolds possessed the highest antiproliferative activity (Shyyka et al., 2019; Pokhodylo et al., 2013, 2014). Furthermore, a series of 6,7-disubstituted-4-(2-fluorophenoxy)quinoline derivatives possessing the 1,2,3-triazole-4carboxamide moiety have been evaluated against $c$-Met kinase and five typical cancer cell lines (A549, H460, HT-29, MKN-45 and U87MG) and exhibited moderate to excellent 
antiproliferative activity (Zhou et al., 2014). A library of 1-benzyl- $N$-(2-(phenylamino)pyridin-3-yl)-1H-1,2,3-triazole4-carboxamides was screened for their antiproliferative activity and showed promising cytotoxicity against lung cancer cell line A549 (Prasad et al., 2019). In addition to the antitumor studies, $1 \mathrm{H}$-1,2,3-triazole-4-carboxamides exhibit other biological activities such as fungicidal (Wang et al., 2014), antiviral (Krajczyk et al., 2014) and antimicrobial (Jadhav et al., 2017) activities and were found to be inhibitors of the $\mathrm{Wnt} / \beta$ catenin signalling pathway (Obianom et al., 2019). It should be noted that the diversity of such compounds can be obtained by amidation of $1 H$-1,2,3-triazole-4-carboxylic acids prepared by convenient Dimroth synthesis and further modifications (Pokhodylo et al., 2009, 2017, 2018; Pokhodylo, Matiychuk et al., 2010; Pokhodylo, Savka et al., 2010; Pokhodylo \& Obushak, 2019). Given the considerable interest in such scaffolds for drug discovery, a detailed study of their structural features is relevant and the crystal structure of the title compound, $\mathrm{C}_{19} \mathrm{H}_{17} \mathrm{ClN}_{4} \mathrm{O}_{2}$, is described herein.<smiles>COc1ccc(-n2nnc(C(=O)Nc3ccc(Cl)cc3)c2C2CC2)cc1</smiles>

\section{Structural commentary}

The title compound crystallizes in the monoclinic centrosymmetric space group $P 2_{1} / n$, with one molecule in the asymmetric unit. As shown in Fig. 1, the 4-methoxyphenyl and 1,2,3-triazole rings are turned relative to each other by $87.77(7)^{\circ}$ because of a significant steric hindrance of the cyclopropyl ring relative to the 4-methoxyphenyl substituent [the $\mathrm{N} 1-\mathrm{C} 9-\mathrm{C} 11-\mathrm{C} 13$ and $\mathrm{N} 1-\mathrm{C} 9-\mathrm{C} 11-\mathrm{C} 12$ torsion angles are $41.2(4)$ and $-31.6(4)^{\circ}$, respectively]. The above angle between the planes is comparable with that for the bulky 5-(2-phenylhydrazineylidene)methyl analogue [73.3 (2) ${ }^{\circ}$; Pokhodylo et al., 2018] but is considerably larger than in the structure of 5-cyclopropyl-1-(3-methoxyphenyl)-1H-1,2,3triazole-4-carboxylic acid [39.1 (2) ${ }^{\circ}$ in which the cyclopropyl ring is oriented to the triazole ring (Pokhodylo et al., 2017) or in 5-methyl-1-(4-nitrophenyl)-1H-1,2,3-triazol-4-ylphosphon-

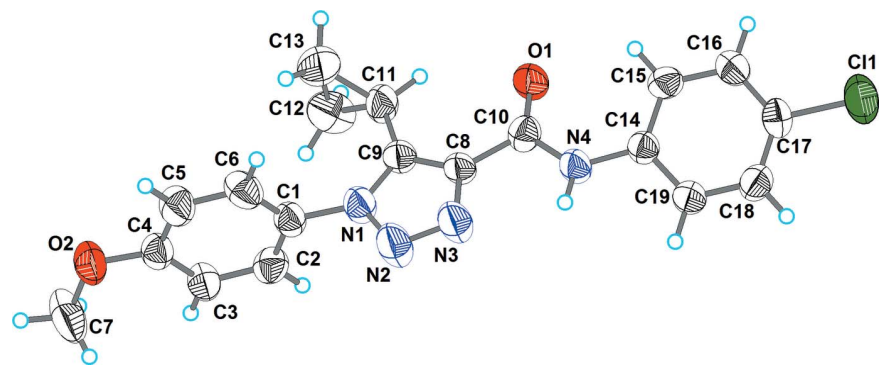

Figure 1

The molecular structure of the title compound with displacement ellipsoids drawn at the $50 \%$ probability level.
Table 1

Hydrogen-bond geometry $\left(\AA,^{\circ}\right)$.

\begin{tabular}{lllll}
\hline$D-\mathrm{H} \cdots A$ & $D-\mathrm{H}$ & $\mathrm{H} \cdots A$ & $D \cdots A$ & $D-\mathrm{H} \cdots A$ \\
\hline $\mathrm{N} 4-\mathrm{H} 4 \cdots \mathrm{N} 3$ & 0.86 & 2.24 & $2.680(3)$ & 112 \\
$\mathrm{~N} 4-\mathrm{H} 4 \cdots \mathrm{N} 2^{\mathrm{i}}$ & 0.86 & 2.68 & $3.491(2)$ & 157 \\
$\mathrm{C} 15-\mathrm{H} 15 \cdots \mathrm{O} 1$ & 0.93 & 2.39 & $2.936(2)$ & 117 \\
$\mathrm{C} 19-\mathrm{H} 19 \cdots \mathrm{N} 2^{\mathrm{i}}$ & 0.93 & 2.68 & $3.475(3)$ & 144 \\
$\mathrm{C} 2-\mathrm{H} 2 \cdots \mathrm{O} 1^{\mathrm{ii}}$ & 0.93 & 2.53 & $3.439(3)$ & 167 \\
$\mathrm{C} 11-\mathrm{H} 11 \cdots \mathrm{O} 1$ & 0.98 & 2.47 & $3.124(2)$ & 124 \\
\hline
\end{tabular}

Symmetry codes: (i) $-x+1,-y,-z+1$; (ii) $-x+1,-y+1,-z+1$.

ate [45.36 (6) ; Pokhodylo et al., 2020]. In selected 5-free triazoles, 1-(3-bromo- or 4-fluorophenyl)-1H-1,2,3-triazol-4yl)methyl methylphosphonates, this angle is 22.9 (3) and 15.7 (2) ${ }^{\circ}$, respectively (Pokhodylo, Shyyka et al., 2019). Within the cyclopropyl ring in the title compound, the three $\mathrm{C}-\mathrm{C}$ bond lengths differ by an insignificant amount $[\mathrm{C} 11-\mathrm{C} 12=$ 1.491 (3), $\mathrm{C} 11-\mathrm{C} 13=1.475$ (3), C12-C13 = 1.457 (3) А]. The amide group is turned slightly by $7.5(3)^{\circ}$ relative to the triazole ring while the proton of the amide group is involved in an intramolecular hydrogen bond with the heterocyclic N3 atom (Table 1). The angle between the 4-chlorophenyl and 1,2,3triazole planes is $29.8(1)^{\circ}$.

\section{Supramolecular features}

As shown in Fig. 2 and Table 2, the extended structure of the title compound is consolidated by a number of intermolecular interactions. Two molecules arranged in a near coplanar manner relative to the triazole ring planes are interconnected by $\mathrm{N} 4-\mathrm{H} 4 \cdots \mathrm{N} 2{ }^{\mathrm{i}}$ and $\mathrm{C} 19-\mathrm{H} 19 \cdots \mathrm{N} 2{ }^{\mathrm{i}}$ hydrogen bonds into a homodimer. Within the dimer, the edge-to-face stacked aromatic rings are tilted by $58.0(3)^{\circ}$. Atom $\mathrm{O} 1$ of the amide group accepts both an intramolecular $\mathrm{C}-\mathrm{H} \cdots \mathrm{O}$ link (with the 4-chlorophenyl and cyclopropyl $\mathrm{H}$ atoms) and an intermolecular $\mathrm{C} 2-\mathrm{H} 2 \cdots \mathrm{O} 1$ interaction with the 4-methoxyphenyl $\mathrm{H}$ atom. The last of these links neighbouring dimers

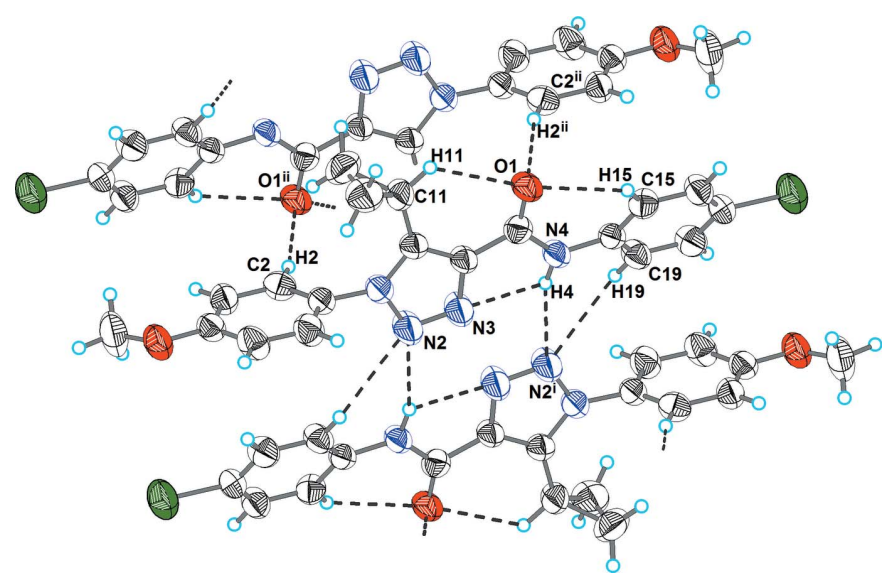

Figure 2

The hydrogen bonding of molecules in the title compound. Hydrogen bonds are shown as dashed lines. The symmetry codes are as in Table 1. 


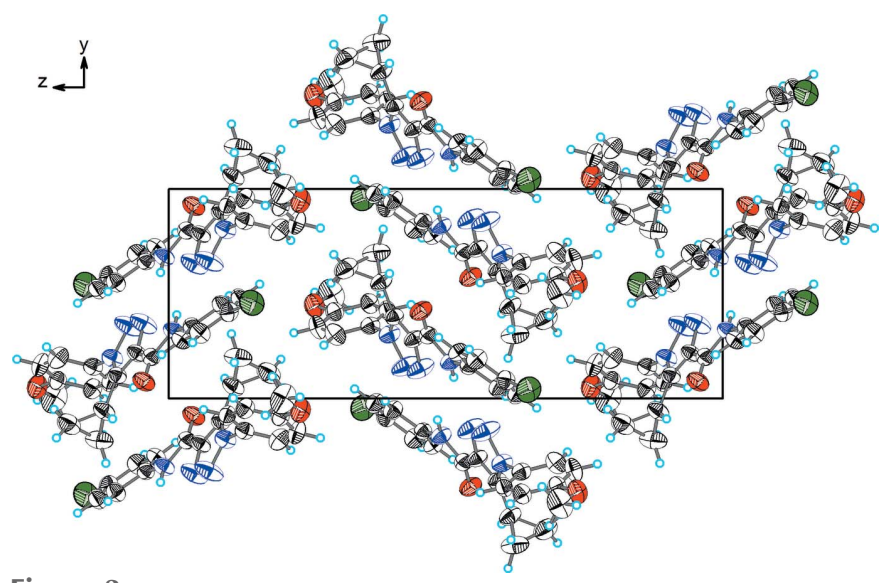

Figure 3

A view along the $a$ axis of the crystal packing of the title compound.

into hydrogen-bonded ribbons parallel to the [010] direction (Fig. 3).

\section{Hirshfeld surface analysis and computational study}

Hirshfeld surface analysis was used to analyse the various intermolecular interactions in the title compound, through mapping the normalized contact distance $\left(d_{\text {norm }}\right)$ using CrystalExplorer (Turner et al., 2017; Spackman \& Jayatilaka, 2009). Hirshfeld surfaces enable the visualization of intermolecular interactions by using different colours and colour intensity to represent short or long contacts and indicate the relative strength of the interactions. The most prominent interactions (the ortho-proton of the aryltriazole moiety and the carbonyl group as well as bifurcated interactions among protons of the amide group and the ortho-proton of the aryl group with the triazole ring nitrogen (N2) atoms of neighbouring molecules) can be seen in the Hirshfeld surface plot as red areas (Fig. 4). Fingerprint plots were produced to show the intermolecular surface bond distances with the regions highlighted for (C) $\mathrm{H} \cdots \mathrm{O}$ and $(\mathrm{C}, \mathrm{N}) \mathrm{H} \cdots \mathrm{N}$ interactions (Fig. 4). The contribution to the surface area for such contacts are $11.6 \%$ and $10.8 \%$, respectively.

The frontier molecular orbitals HOMO and LUMO were analysed to better understand the electronic charge transfer within the molecule and its electron donating and accepting

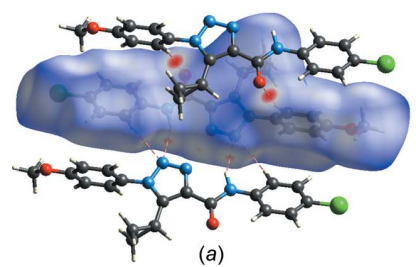

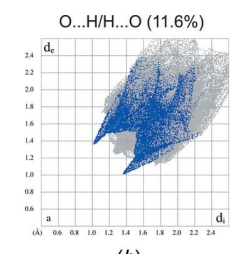

(b)

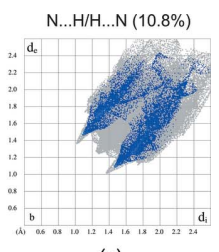

(c)
Figure 4

(a) Hirshfeld surface for the title molecule mapped with $d_{\text {norm }}$ over the range -0.171 to 1.473 a.u. showing $\mathrm{N}-\mathrm{H} \cdots \mathrm{N}, \mathrm{C}-\mathrm{H} \cdots \mathrm{N}$ and $\mathrm{C}-\mathrm{H} \cdots \mathrm{O}$ hydrogen-bonded contacts. Fingerprint plots resolved into $(b) \mathrm{N} \cdots \mathrm{H} /$ $\mathrm{H} \cdots \mathrm{N}$ and $(c) \mathrm{O} \cdots \mathrm{H} / \mathrm{H} \cdots \mathrm{O}$ contacts. Neighbouring molecules associated with close contacts are also shown.

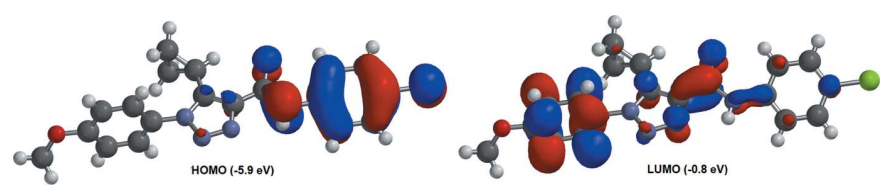

Figure 5

Frontier molecular orbital energies.

ability. The molecular orbital energies were calculated using the B3LYP functional level with the $6-31+\mathrm{G}^{*}$ basis set in a vacuum with GAMESS software (Schmidt et al., 1993). The HOMO and LUMO orbitals were found to be well separated in energy and largely localized on the 4-chlorophenyl amide or aryltriazole motifs, respectively (Fig. 5). Their respective energy values were estimated to be $-5.9 \mathrm{eV}$ and $-0.8 \mathrm{eV}$.

\section{Database survey}

The closest related compounds containing a similar 1-aryl-1H1,2,3-triazole-4-carboxamide skeleton to the title compound but with different substituents on the amide are: $(S)-1-(4-$ chlorophenyl)- $N$-(-1-hydroxy-3-phenylpropan-2-yl)-5-methyl-1H-1,2,3-triazole-4-carboxamide (I) (CCDC refcode: ZIPSEY; Shen et al., 2013), 1-(4-chlorophenyl)-5-methyl- $N$ [(3-phenyl-1,2-oxazol-5-yl)methyl]-1H-1,2,3-triazole-4-carboxamide (II) (LELHOB; Niu et al., 2013), (5-methyl-1-(8[trifluoromethyl)quinolin-4-yl]-1H-1,2,3-triazol-4-yl)morpholino)methanone (III) (LOHWIP; Anuradha et al., 2008) and 1-(3-amino-5-(3-hydroxy-3-methylbut-1-yn-1-yl)phenyl)- $N$ butyl-1H-1,2,3-triazole-4-carboxamide (IV) (BEBJEZ; Li et al., 2012).

Compounds (I) and (II) crystallize in the monoclinic crystal system [non-centrosymmetric space group $P 2_{1}$ in (I) and centrosymmetric $P 2_{1} / \mathrm{c}$ in (II)], while compounds (III) and (IV) crystallize in the triclinic space group $P \overline{1}$. Structure (I) contains two crystallographically independent molecules, the hydroxyl groups of which participate in intermolecular $\mathrm{O}-$ $\mathrm{H}$... O hydrogen bonds. In contrast to the structure of title compound, the dihedral angles between the phenyl rings and triazole rings in (I) are $-45.2(6)^{\circ}(\mathrm{C} 5-\mathrm{C} 6-\mathrm{N} 1-\mathrm{N} 2)$ and $39.9(6)^{\circ}\left(\mathrm{C1}^{\prime}-\mathrm{C}^{\prime}-\mathrm{N} 1^{\prime}-\mathrm{N} 2^{\prime}\right)$. The analogous angle in (II) is $19.2(2)^{\circ}$. In structure (II), the carboxamide groups connect neighbouring molecules into infinite hydrogen-bonded chains by means of $\mathrm{N}-\mathrm{H} \cdots \mathrm{O}$ hydrogen bonds: these are linked by $\mathrm{N}-\mathrm{H}$... (oxazole) contacts into a three-dimensional framework. Similarly to (I) and (II), structure (III) contains a 5-methyl substituent at the triazole ring and, because of significant steric hindrance of the 8-(trifluoromethyl)quinoline group, the dihedral angle between the rings is $54.7^{\circ}$. The phenyl and triazole rings in (IV) are close to coplanar $\left(7.5^{\circ}\right)$, while the hydroxyl, carboxamide and amino groups participate in $\mathrm{O}-\mathrm{H} \cdots \mathrm{O}$ and $\mathrm{N}-\mathrm{H} \cdots \mathrm{O}$ hydrogen bonds. Finally, two copper(I) $\pi$-complexes with compositions $\left[\mathrm{Cu}\left(\mathrm{C}_{12} \mathrm{H}_{13} \mathrm{~N}_{5} \mathrm{O}\right)\right.$ $\left.\left(\mathrm{NO}_{3}\right)\right] \cdot 0.5 \mathrm{H}_{2} \mathrm{O}$ and $\left[\mathrm{Cu}\left(\mathrm{C}_{12} \mathrm{H}_{13} \mathrm{~N}_{5} \mathrm{O}\right)\left(\mathrm{CF}_{3} \mathrm{COO}\right)\right]\left(\mathrm{C}_{12} \mathrm{H}_{13} \mathrm{~N}_{5} \mathrm{O}\right.$ is $\mathrm{N}$-allyl-5-amino-1-phenyl-1 $\mathrm{H}$-1,2,3-triazole-4-carboxamide) were obtained by electrochemical synthesis (ZEQTOG and ZEQTUM; Slyvka et al., 2012). Crystals of both compounds 


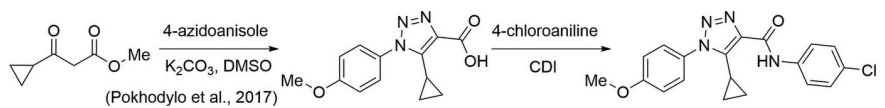

Figure 6

Synthesis of $\mathrm{N}$-(4-chlorophenyl)-5-cyclopropyl-1-(4-methoxyphenyl)-1H1,2,3-triazole-4-carboxamide.

are monoclinic, space group $C 2 / c$. In both structures, the $N$-allyl-1H-1,2,3-triazole-4-carboxamide moiety acts as a bridging chelating ligand and forms, with the copper(I) atoms, infinite chains containing $\left[\mathrm{CuC}_{4} \mathrm{NO}\right]$ seven-membered rings.

\section{Synthesis and crystallization}

The title compound was synthesized from 5-cyclopropyl-1-(4methoxyphenyl)-1H-1,2,3-triazole-4-carboxylic acid (Pokhodylo et al., 2017) by the following procedure (Fig. 6). 5-Cyclopropyl-1-(4-methoxyphenyl)-1H-1,2,3-triazole-4-carboxylic acid $1(1.3 \mathrm{~g}, 5.0 \mathrm{mmol})$ was added to a solution of $1,1^{\prime}$ carbonyldiimidazole $(0.81 \mathrm{~g}, 5.0 \mathrm{mmol})$ in dry acetonitrile $(25 \mathrm{ml})$ and the mixture was kept for $30 \mathrm{~min}$ at $323 \mathrm{~K}$. Then $4-$ chloroaniline $2(0.64 \mathrm{~g}, 5.0 \mathrm{mmol})$ was added, and the mixture was heated at $343 \mathrm{~K}$ for $1 \mathrm{~h}$. After cooling to room temperature, water $(30 \mathrm{ml})$ was added. The precipitate was filtered off, washed with water on a filter, recrystallized from ethanol solution, and dried in air to give the title compound as colourless prismatic crystals, m.p. $422-423 \mathrm{~K} ;{ }^{1} \mathrm{H}$ NMR $\left(500 \mathrm{MHz}, \mathrm{DMSO}-d_{6}\right) \delta 10.56(s, 1 \mathrm{H}, \mathrm{NH}), 7.89(d, J=8.6 \mathrm{~Hz}$, $\left.2 \mathrm{H}, \mathrm{H}_{\mathrm{Ar}}\right), 7.58\left(d, J=8.6 \mathrm{~Hz}, 2 \mathrm{H}, \mathrm{H}_{\mathrm{Ar}}\right), 7.39(d, J=8.6 \mathrm{~Hz}, 2 \mathrm{H}$, $\left.\mathrm{H}_{\mathrm{Ar}}\right), 7.16\left(d, J=8.6 \mathrm{~Hz}, 2 \mathrm{H}, \mathrm{H}_{\mathrm{Ar}}\right), 3.86(s, 3 \mathrm{H}, \mathrm{MeO}), 2.10$ $1.99\left(m, 1 \mathrm{H},{ }_{\mathrm{cPr}} \mathrm{CH}\right), 0.95-0.80\left(m, 4 \mathrm{H},{ }_{\mathrm{cPr}} \mathrm{CH}_{2}\right) ;{ }^{13} \mathrm{C} \mathrm{NMR}$ $\left(126 \mathrm{MHz}, \mathrm{DMSO}-d_{6}\right) \delta 160.62\left(\mathrm{C}=\mathrm{O}\right.$ or $\left.\mathrm{C}_{\mathrm{Ar}}-\mathrm{O}\right), 159.60$ $\left(\mathrm{C}=\mathrm{O}\right.$ or $\left.\mathrm{C}_{\mathrm{Ar}}-\mathrm{O}\right), 142.26\left(\mathrm{C}_{\text {Triazole }}-4\right), 138.87\left(\mathrm{C}_{\text {Triazole }}-5\right)$, 138.21 ( $\left.\mathrm{C}^{\mathrm{ClAr}}-1\right), 129.08\left(\mathrm{C}_{\mathrm{Ar}}-1\right), 128.91\left(2 \times \mathrm{C}_{\mathrm{ClAr}}-3,5\right), 127.77$ $\left(2 \times \mathrm{C}_{\mathrm{Ar}}-2,6\right), 127.70\left(\mathrm{C}_{\mathrm{ClAr}}-4\right), 122.25\left(2 \times \mathrm{C}_{\mathrm{ClAr}}-2,6\right), 115.00$ $\left(2 \times \mathrm{C}_{\mathrm{Ar}^{-}}{ }^{-3,5}\right), 56.06(\mathrm{MeO}), 8.09\left(2 \times \mathrm{CH}_{2}{ }^{\mathrm{cPr}}\right), 5.75\left(\mathrm{CH}^{\mathrm{cPr}}\right)$; MS $m / z=369\left(M^{+}+1\right)$; Analysis calculated for $\mathrm{C}_{19} \mathrm{H}_{17} \mathrm{ClN}_{4} \mathrm{O}_{2}$ $\left(M_{\mathrm{r}}=368.82\right),(\%): \mathrm{C} 61.88, \mathrm{H} 4.65, \mathrm{~N} 15.19$; found $(\%)$ : $\mathrm{C}$ 61.91, H 4.74, N 15.21.

\section{Refinement}

Crystal data, data collection and structure refinement details are summarized in Table 2. All $\mathrm{H}$ atoms were positioned geometrically with $\mathrm{N}-\mathrm{H}=0.86 \AA$ and $\mathrm{C}-\mathrm{H}=0.93-0.98 \AA$ and refined as riding atoms. The constraint $U_{\text {iso }}(\mathrm{H})=$ $1.2 U_{\text {eq }}$ (carrier) or $1.5 U_{\text {eq }}(\mathrm{C}$-methyl carrier $)$ was applied in all cases.

\section{Funding information}

The authors are grateful to the Ministry of Education and Science of Ukraine for financial support of this project.

\section{References}

Anuradha, N., Thiruvalluvar, A., Mahalinga, M. \& Butcher, R. J. (2008). Acta Cryst. E64, o2375.
Table 2

Experimental details.

\begin{tabular}{|c|c|}
\hline \multicolumn{2}{|l|}{ Crystal data } \\
\hline Chemical formula & $\mathrm{C}_{19} \mathrm{H}_{17} \mathrm{ClN}_{4} \mathrm{O}_{2}$ \\
\hline$M_{\mathrm{r}}$ & 368.82 \\
\hline Crystal system, space group & Monoclinic, $P 2_{1} / n$ \\
\hline Temperature $(\mathrm{K})$ & 293 \\
\hline$a, b, c(\AA)$ & $\begin{array}{l}10.5673(4), 8.0182(3) \\
21.2318(10)\end{array}$ \\
\hline$\beta\left(^{\circ}\right)$ & $95.282(4)$ \\
\hline$V\left(\AA^{3}\right)$ & $1791.35(13)$ \\
\hline$Z$ & 4 \\
\hline Radiation type & Мо $K \alpha$ \\
\hline$\mu\left(\mathrm{mm}^{-1}\right)$ & 0.24 \\
\hline Crystal size $(\mathrm{mm})$ & $0.5 \times 0.08 \times 0.07$ \\
\hline \multicolumn{2}{|l|}{ Data collection } \\
\hline Diffractometer & Oxford Diffraction Xcalibur3 CCD \\
\hline Absorption correction & $\begin{array}{l}\text { Multi-scan (CrysAlis RED; Oxford } \\
\text { Diffraction, 2005) }\end{array}$ \\
\hline$T_{\min }, T_{\max }$ & $0.890,0.982$ \\
\hline $\begin{array}{l}\text { No. of measured, independent and } \\
\text { observed }[I>2 \sigma(I)] \text { reflections }\end{array}$ & $10913,3475,1534$ \\
\hline$R_{\text {int }}$ & 0.046 \\
\hline$(\sin \theta / \lambda)_{\max }\left(\AA^{-1}\right)$ & 0.617 \\
\hline \multicolumn{2}{|l|}{ Refinement } \\
\hline$R\left[F^{2}>2 \sigma\left(F^{2}\right)\right], w R\left(F^{2}\right), S$ & $0.040,0.053,1.05$ \\
\hline No. of reflections & 3475 \\
\hline No. of parameters & 236 \\
\hline $\mathrm{H}$-atom treatment & H-atom parameters constrained \\
\hline$\Delta \rho_{\max }, \Delta \rho_{\min }\left(\mathrm{e} \AA^{-3}\right)$ & $0.14,-0.19$ \\
\hline
\end{tabular}

Computer programs: CrysAlis PRO (Oxford Diffraction, 2005), SHELXT (Sheldrick, 2015a), SHELXL (Sheldrick, 2015b) and OLEX2 (Dolomanov et al., 2009).

Bonnefond, M., Florent, R., Lenoir, S., Lambert, B., Abeilard, E., Giffard, F., Louis, M., Elie, N., Briand, M., Vivien, D., Poulain, L., Gauduchon, P. \& N'Diaye, M. (2018). Oncotarget, 9, 3389633911.

Dolomanov, O. V., Bourhis, L. J., Gildea, R. J., Howard, J. A. K. \& Puschmann, H. (2009). J. Appl. Cryst. 42, 339-341.

Elamari, H., Slimi, R., Chabot, G. G., Quentin, L., Scherman, D. \& Girard, C. (2013). Eur. J. Med. Chem. 60, 360-364.

Figg, W. D., Cole, K. A., Reed, E., Steinberg, S. M., Piscitelli, S. C., Davis, P. A., Soltis, M. J., Jacob, J., Boudoulas, S. \& Goldspiel, B. (1995). Clin. Cancer Res. 1, 797-803.

Jadhav, R. P., Raundal, H. N., Patil, A. A. \& Bobade, V. D. (2017). J. Saudi Chem. Soc. 21, 152-159.

Krajczyk, A., Kulinska, K., Kulinski, T., Hurst, B. L., Day, C. W., Smee, D. F., Ostrowski, T., Januszczyk, P. \& Zeidler, J. (2014). Antivir. Chem. Chemother. 23, 161-171.

Li, Y.-J., Xu, L., Yang, W.-L., Liu, H.-B., Lai, S.-W., Che, C.-M. \& Li, Y.-L. (2012). Chem. Eur. J. 18, 4782-4790.

Niu, T.-F., Lv, M.-F., Wang, L., Yi, W.-B. \& Cai, C. (2013). Org. Biomol. Chem. 11, 1040-1048.

Obianom, O. N., Ai, Y., Li, Y., Yang, W., Guo, D., Yang, H., Sakamuru, S., Xia, M., Xue, F. \& Shu, Y. (2019). J. Med. Chem. 62, 727-741.

Oxford Diffraction (2005). CrysAlis PRO. Oxford Diffraction, Abingdon, England.

Pokhodylo, N. T., Matiychuk, V. S. \& Obushak, M. D. (2010). Synth. Commun. 40, 1932-1938.

Pokhodylo, N. T. \& Obushak, M. D. (2019). Russ. J. Org. Chem. 55, 1241-1243.

Pokhodylo, N. T., Savka, R. D., Pidlypnyi, N. I., Matiychuk, V. S. \& Obushak, M. D. (2010). Synth. Commun. 40, 391-399.

Pokhodylo, N. T., Shyyka, O. Ya., Goreshnik, E. A. \& Obushak, M. D. (2020). ChemistrySelect, 5, 260-264.

Pokhodylo, N. T., Shyyka, O. Ya. \& Matiychuk, V. S. (2014). Med. Chem. Res. 23, 2426-2438. 
Pokhodylo, N. T., Shyyka, O. Ya., Matiychuk, V. S., Obushak, M. D. \& Pavlyuk, V. V. (2017). ChemistrySelect, 2, 5871-5876.

Pokhodylo, N. T., Shyyka, O. Ya. \& Obushak, M. D. (2018). Chem. Heterocycl. Compd, 54, 773-779.

Pokhodylo, N. T., Shyyka, O. Ya., Skrobala, V. E. \& Matiychuk, V. S. (2013). Clinical Pharmacy, Pharmacotherapy \& Medical Standardization, Vol. 16-17, pp. 92-97. (In Ukrainian) http://nbuv.gov.ua/ UJRN/Kff_2012_3_15

Pokhodylo, N. T., Shyyka, O. Ya., Tupychak, M. A., Slyvka, Yu. I. \& Obushak, M. D. (2019). Chem. Heterocycl. C. 55, 374-378.

Pokhodylo, N. T., Teslenko, Y. O., Matiychuk, V. S. \& Obushak, M. D. (2009). Synthesis, pp. 2741-2748.

Prasad, B., Lakshma Nayak, V., Srikanth, P. S., Baig, M. F., Subba Reddy, N. V., Babu, K. S. \& Kamal, A. (2019). Bioorg. Chem. 83, 535-548.

Schmidt, M. W., Baldridge, K. K., Boatz, J. A., Elbert, S. T., Gordon, M. S., Jensen, J. H., Koseki, S., Matsunaga, N., Nguyen, K. A., Su, S. J., Windus, T. L., Dupuis, M. \& Montgomery, J. A. (1993). J. Comput. Chem. 14, 1347-1363.
Sheldrick, G. M. (2015a). Acta Cryst. A71, 3-8.

Sheldrick, G. M. (2015b). Acta Cryst. C71, 3-8.

Shen, G.-L., Chen, Z.-B., Wu, Z.-F. \& Dong, H.-S. (2013). J. Heterocycl. Chem. 50, 781-786.

Shyyka, O. Ya., Pokhodylo, N. T. \& Finiuk, N. S. (2019). Biopolym. Cell, 35, 321-330.

Slyvka, Yu. I., Pavlyuk, A. V., Ardan, B. R., Pokhodilo, N. T., Goreshnik, E. A. \& Demchenko, P. Yu. (2012). Russ. J. Inorg. Chem. 57, 815-821.

Spackman, M. A. \& Jayatilaka, D. (2009). CrystEngComm, 11, 19-32. Turner, M. J., Mckinnon, J. J., Wolff, S. K., Grimwood, D. J., Spackman, P. R., Jayatilaka, D. \& Spackman, M. A. (2017). CrystalExplorer17. The University of Western Australia. http:// hirshfeldsurface.net

Wang, Z., Gao, Y., Hou, Y., Zhang, C., Yu, S. J., Bian, Q., Li, Z. M. \& Zhao, W. G. (2014). Eur. J. Med. Chem. 86, 87-94.

Wheless, J. W. \& Vazquez, B. (2010). Epilepsy Curr. 10, 1-6.

Zhou, S., Liao, H., Liu, M., Feng, G., Fu, B., Li, R., Cheng, M., Zhao, Y. \& Gong, P. (2014). Bioorg. Med. Chem. 22, 6438-6452. 


\section{supporting information}

Acta Cryst. (2020). E76, 756-760 [https://doi.org/10.1107/S2056989020005848]

Synthesis, crystal structure and Hirshfeld surface analysis of $\mathrm{N}$-(4-chlorophenyl)-5-cyclopropyl-1-(4-methoxyphenyl)-1H-1,2,3-triazole-4-carboxamide

\section{Nazariy Pokhodylo, Yurii Slyvka and Volodymyr Pavlyuk}

Computing details

Data collection: CrysAlis PRO (Oxford Diffraction, 2005); cell refinement: CrysAlis PRO (Oxford Diffraction, 2005); data reduction: CrysAlis PRO (Oxford Diffraction, 2005); program(s) used to solve structure: ShelXT (Sheldrick, 2015a); program(s) used to refine structure: SHELXL (Sheldrick, 2015b); molecular graphics: OLEX2 (Dolomanov et al., 2009); software used to prepare material for publication: OLEX2 (Dolomanov et al., 2009).

N-(4-Chlorophenyl)-5-cyclopropyl-1-(4-methoxyphenyl)-1H-1,2,3-triazole-4-carboxamide

\section{Crystal data}

$\mathrm{C}_{19} \mathrm{H}_{17} \mathrm{ClN}_{4} \mathrm{O}_{2}$

$M_{r}=368.82$

Monoclinic, $P 2{ }_{1} / n$

$a=10.5673$ (4) $\AA$

$b=8.0182(3) \AA$

$c=21.2318(10) \AA$

$\beta=95.282(4)^{\circ}$

$V=1791.35(13) \AA^{3}$

$Z=4$

Data collection

Oxford Diffraction Xcalibur3 CCD diffractometer

$\omega$ scans

Absorption correction: multi-scan

(CrysAlis RED; Oxford Diffraction, 2005)

$T_{\text {min }}=0.890, T_{\max }=0.982$

10913 measured reflections

\section{Refinement}

Refinement on $F^{2}$

Least-squares matrix: full

$R\left[F^{2}>2 \sigma\left(F^{2}\right)\right]=0.040$

$w R\left(F^{2}\right)=0.053$

$S=1.05$

3475 reflections

236 parameters

0 restraints
$F(000)=768$

$D_{\mathrm{x}}=1.368 \mathrm{Mg} \mathrm{m}^{-3}$

Mo $K \alpha$ radiation, $\lambda=0.71073 \AA$

Cell parameters from 1540 reflections

$\theta=0.9-1.0^{\circ}$

$\mu=0.24 \mathrm{~mm}^{-1}$

$T=293 \mathrm{~K}$

Prism, colourless

$0.5 \times 0.08 \times 0.07 \mathrm{~mm}$

3475 independent reflections

1534 reflections with $I>2 \sigma(I)$

$R_{\text {int }}=0.046$

$\theta_{\max }=26.0^{\circ}, \theta_{\min }=2.7^{\circ}$

$h=-12 \rightarrow 12$

$k=-5 \rightarrow 9$

$l=-25 \rightarrow 26$

Hydrogen site location: inferred from neighbouring sites

$\mathrm{H}$-atom parameters constrained

$w=1 /\left[\sigma^{2}\left(F_{\mathrm{o}}^{2}\right)+(0.0071 P)^{2}+0.050 P\right]$

where $P=\left(F_{\mathrm{o}}^{2}+2 F_{\mathrm{c}}^{2}\right) / 3$

$(\Delta / \sigma)_{\max }=0.001$

$\Delta \rho_{\max }=0.14 \mathrm{e} \AA^{-3}$

$\Delta \rho_{\min }=-0.19 \mathrm{e}^{-3}$ 


\section{Special details}

Geometry. All esds (except the esd in the dihedral angle between two 1.s. planes) are estimated using the full covariance matrix. The cell esds are taken into account individually in the estimation of esds in distances, angles and torsion angles; correlations between esds in cell parameters are only used when they are defined by crystal symmetry. An approximate (isotropic) treatment of cell esds is used for estimating esds involving l.s. planes.

Fractional atomic coordinates and isotropic or equivalent isotropic displacement parameters $\left(\AA^{2}\right)$

\begin{tabular}{|c|c|c|c|c|}
\hline & $x$ & $y$ & $z$ & $U_{\text {iso }} * / U_{\text {eq }}$ \\
\hline $\mathrm{Cl1}$ & $1.19945(6)$ & $0.04278(8)$ & $0.35044(3)$ & $0.0860(2)$ \\
\hline $\mathrm{O} 1$ & $0.80102(13)$ & $0.41861(18)$ & $0.54511(7)$ & $0.0627(5)$ \\
\hline $\mathrm{O} 2$ & $0.01792(15)$ & $0.44313(19)$ & $0.73741(7)$ & $0.0686(5)$ \\
\hline N4 & $0.75389(15)$ & $0.1763(2)$ & $0.49150(8)$ & $0.0524(5)$ \\
\hline $\mathrm{H} 4$ & 0.695819 & 0.101374 & 0.485272 & $0.063 *$ \\
\hline N1 & $0.43143(16)$ & $0.3069(2)$ & $0.60195(9)$ & $0.0556(5)$ \\
\hline $\mathrm{C} 14$ & $0.8626(2)$ & $0.1540(2)$ & $0.45845(11)$ & $0.0443(6)$ \\
\hline $\mathrm{C} 1$ & $0.3243(2)$ & $0.3477(2)$ & $0.63661(12)$ & $0.0492(6)$ \\
\hline C9 & $0.5403(2)$ & $0.3903(2)$ & $0.59331(10)$ & $0.0479(6)$ \\
\hline $\mathrm{C} 4$ & $0.1154(2)$ & $0.4142(3)$ & $0.70082(12)$ & $0.0512(6)$ \\
\hline $\mathrm{C} 10$ & $0.7294(2)$ & $0.3016(3)$ & $0.53206(11)$ & $0.0505(6)$ \\
\hline N3 & $0.53626(18)$ & $0.1428(2)$ & $0.54590(10)$ & $0.0763(7)$ \\
\hline $\mathrm{C} 15$ & $0.9796(2)$ & $0.2218(2)$ & $0.47738(10)$ & $0.0520(6)$ \\
\hline H15 & 0.989197 & 0.290445 & 0.512804 & $0.062 *$ \\
\hline $\mathrm{C} 8$ & $0.6050(2)$ & $0.2842(3)$ & $0.55768(11)$ & $0.0486(6)$ \\
\hline $\mathrm{C} 3$ & $0.11310(19)$ & $0.4532(2)$ & $0.63777(11)$ & $0.0546(6)$ \\
\hline H3 & 0.041147 & 0.501519 & 0.616770 & $0.066^{*}$ \\
\hline N2 & 0.43009 (18) & 0.1551 (2) & $0.57229(11)$ & $0.0831(7)$ \\
\hline C19 & $0.84942(19)$ & $0.0551(3)$ & $0.40452(10)$ & $0.0539(6)$ \\
\hline H19 & 0.770296 & 0.011077 & 0.390662 & $0.065^{*}$ \\
\hline $\mathrm{C} 2$ & $0.2188(2)$ & 0.4199 (3) & $0.60555(10)$ & $0.0546(6)$ \\
\hline $\mathrm{H} 2$ & 0.218048 & 0.446667 & 0.562890 & $0.066^{*}$ \\
\hline $\mathrm{C} 16$ & $1.0831(2)$ & $0.1881(3)$ & $0.44386(11)$ & $0.0573(7)$ \\
\hline H16 & 1.162198 & 0.233351 & 0.456901 & $0.069 *$ \\
\hline C11 & $0.57821(19)$ & $0.5584(3)$ & $0.61661(11)$ & $0.0596(6)$ \\
\hline H11 & 0.658079 & 0.596910 & 0.601492 & $0.072 *$ \\
\hline $\mathrm{C} 18$ & $0.9525(2)$ & $0.0218(2)$ & $0.37150(10)$ & $0.0585(7)$ \\
\hline H18 & 0.943421 & -0.045418 & 0.335686 & $0.070 *$ \\
\hline $\mathrm{C} 17$ & $1.0685(2)$ & $0.0881(3)$ & $0.39158(11)$ & $0.0533(6)$ \\
\hline $\mathrm{C} 5$ & $0.2213(2)$ & $0.3398(3)$ & $0.73129(11)$ & $0.0635(7)$ \\
\hline H5 & 0.222148 & 0.312257 & 0.773873 & $0.076^{*}$ \\
\hline C6 & $0.3263(2)$ & $0.3056(3)$ & $0.69943(12)$ & $0.0623(7)$ \\
\hline H6 & 0.397430 & 0.254642 & 0.720160 & $0.075^{*}$ \\
\hline $\mathrm{C} 13$ & $0.5580(2)$ & $0.6216(3)$ & $0.68014(12)$ & $0.0782(8)$ \\
\hline H13A & 0.511849 & 0.550992 & 0.707204 & $0.094 *$ \\
\hline H13B & 0.625552 & 0.687084 & 0.702118 & $0.094 *$ \\
\hline C12 & $0.4869(2)$ & 0.6965 (3) & $0.62524(13)$ & $0.0809(8)$ \\
\hline $\mathrm{H} 12 \mathrm{~A}$ & 0.510672 & 0.808059 & 0.613084 & $0.097 *$ \\
\hline H12B & 0.396882 & 0.671863 & 0.618174 & $0.097^{*}$ \\
\hline
\end{tabular}




\begin{tabular}{lllll} 
C7 & $-0.0993(2)$ & $0.4984(3)$ & $0.70643(12)$ & $0.0970(9)$ \\
H7A & -0.128382 & 0.419658 & 0.674241 & $0.146^{*}$ \\
H7B & -0.087613 & 0.605304 & 0.687433 & $0.146^{*}$ \\
H7C & -0.161144 & 0.507674 & 0.736641 & $0.146^{*}$ \\
\hline
\end{tabular}

Atomic displacement parameters $\left(\AA^{2}\right)$

\begin{tabular}{lllllll}
\hline & $U^{11}$ & $U^{22}$ & $U^{33}$ & $U^{12}$ & $U^{13}$ & $U^{23}$ \\
\hline C11 & $0.0723(5)$ & $0.0988(5)$ & $0.0925(5)$ & $0.0004(4)$ & $0.0379(4)$ & $-0.0087(4)$ \\
O1 & $0.0573(10)$ & $0.0568(10)$ & $0.0765(12)$ & $-0.0187(8)$ & $0.0189(9)$ & $-0.0171(9)$ \\
O2 & $0.0585(11)$ & $0.0879(12)$ & $0.0627(12)$ & $0.0026(9)$ & $0.0231(10)$ & $0.0082(9)$ \\
N4 & $0.0475(12)$ & $0.0478(12)$ & $0.0639(14)$ & $-0.0110(9)$ & $0.0167(11)$ & $-0.0108(10)$ \\
N1 & $0.0512(13)$ & $0.0499(12)$ & $0.0682(15)$ & $-0.0072(11)$ & $0.0186(12)$ & $-0.0094(11)$ \\
C14 & $0.0454(15)$ & $0.0413(14)$ & $0.0468(15)$ & $-0.0044(11)$ & $0.0087(14)$ & $-0.0008(12)$ \\
C1 & $0.0468(16)$ & $0.0458(14)$ & $0.0565(18)$ & $-0.0048(12)$ & $0.0130(15)$ & $-0.0036(13)$ \\
C9 & $0.0480(15)$ & $0.0428(14)$ & $0.0534(16)$ & $-0.0059(12)$ & $0.0067(14)$ & $-0.0038(12)$ \\
C4 & $0.0516(17)$ & $0.0527(15)$ & $0.0507(17)$ & $-0.0039(12)$ & $0.0127(15)$ & $0.0017(13)$ \\
C10 & $0.0562(17)$ & $0.0463(15)$ & $0.0501(16)$ & $0.0002(13)$ & $0.0103(15)$ & $-0.0024(13)$ \\
N3 & $0.0591(14)$ & $0.0633(15)$ & $0.1115(19)$ & $-0.0171(11)$ & $0.0347(14)$ & $-0.0363(12)$ \\
C15 & $0.0512(15)$ & $0.0488(15)$ & $0.0553(18)$ & $-0.0033(12)$ & $0.0013(15)$ & $-0.0112(12)$ \\
C8 & $0.0436(15)$ & $0.0443(15)$ & $0.0589(17)$ & $-0.0099(12)$ & $0.0110(14)$ & $-0.0123(12)$ \\
C3 & $0.0493(15)$ & $0.0617(15)$ & $0.0540(17)$ & $0.0043(12)$ & $0.0109(14)$ & $0.0055(13)$ \\
N2 & $0.0665(16)$ & $0.0618(14)$ & $0.127(2)$ & $-0.0215(11)$ & $0.0420(15)$ & $-0.0392(13)$ \\
C19 & $0.0503(15)$ & $0.0561(14)$ & $0.0564(16)$ & $-0.0130(12)$ & $0.0109(14)$ & $-0.0093(13)$ \\
C2 & $0.0619(17)$ & $0.0589(15)$ & $0.0434(15)$ & $-0.0041(14)$ & $0.0065(15)$ & $0.0055(12)$ \\
C16 & $0.0456(16)$ & $0.0629(16)$ & $0.0644(19)$ & $-0.0073(13)$ & $0.0105(15)$ & $-0.0063(14)$ \\
C11 & $0.0580(16)$ & $0.0533(15)$ & $0.0704(18)$ & $-0.0040(13)$ & $0.0210(14)$ & $-0.0185(14)$ \\
C18 & $0.0657(17)$ & $0.0595(16)$ & $0.0520(16)$ & $-0.0095(14)$ & $0.0143(15)$ & $-0.0114(12)$ \\
C17 & $0.0523(16)$ & $0.0561(15)$ & $0.0542(17)$ & $0.0004(13)$ & $0.0198(14)$ & $0.0034(13)$ \\
C5 & $0.0602(18)$ & $0.0838(18)$ & $0.0474(17)$ & $-0.0001(14)$ & $0.0095(16)$ & $0.0168(14)$ \\
C6 & $0.0478(17)$ & $0.0717(17)$ & $0.067(2)$ & $0.0024(13)$ & $0.0025(16)$ & $0.0143(15)$ \\
C13 & $0.083(2)$ & $0.0669(18)$ & $0.085(2)$ & $-0.0152(15)$ & $0.0110(19)$ & $-0.0197(16)$ \\
C12 & $0.073(2)$ & $0.0489(16)$ & $0.119(2)$ & $0.0045(14)$ & $-0.0003(19)$ & $-0.0099(17)$ \\
C7 & $0.0580(18)$ & $0.136(3)$ & $0.102(2)$ & $0.0258(17)$ & $0.0314(17)$ & $0.0219(19)$ \\
& & & & & & \\
\hline & & & & & &
\end{tabular}

Geometric parameters $\left(\hat{A},{ }^{o}\right)$

\begin{tabular}{llll}
\hline $\mathrm{C} 11-\mathrm{C} 17$ & $1.742(2)$ & $\mathrm{C} 3-\mathrm{H} 3$ & 0.9300 \\
$\mathrm{O} 1-\mathrm{C} 10$ & $1.221(2)$ & $\mathrm{C} 3-\mathrm{C} 2$ & $1.389(3)$ \\
$\mathrm{O} 2-\mathrm{C} 4$ & $1.366(2)$ & $\mathrm{C} 19-\mathrm{H} 19$ & 0.9300 \\
$\mathrm{O} 2-\mathrm{C} 7$ & $1.419(2)$ & $\mathrm{C} 19-\mathrm{C} 18$ & $1.375(3)$ \\
$\mathrm{N} 4-\mathrm{H} 4$ & 0.8600 & $\mathrm{C} 2-\mathrm{H} 2$ & 0.9300 \\
$\mathrm{~N} 4-\mathrm{C} 14$ & $1.412(2)$ & $\mathrm{C} 16-\mathrm{H} 16$ & 0.9300 \\
$\mathrm{~N} 4-\mathrm{C} 10$ & $1.364(2)$ & $\mathrm{C} 16-\mathrm{C} 17$ & $1.367(3)$ \\
$\mathrm{N} 1-\mathrm{C} 1$ & $1.443(2)$ & $\mathrm{C} 11-\mathrm{H} 11$ & 0.9800 \\
$\mathrm{~N} 1-\mathrm{C} 9$ & $1.358(2)$ & $\mathrm{C} 11-\mathrm{C} 13$ & $1.475(3)$ \\
$\mathrm{N} 1-\mathrm{N} 2$ & $1.370(2)$ & $\mathrm{C} 11-\mathrm{C} 12$ & $1.491(3)$ \\
$\mathrm{C} 14-\mathrm{C} 15$ & $1.376(3)$ & $\mathrm{C} 18-\mathrm{H} 18$ & 0.9300
\end{tabular}




\begin{tabular}{|c|c|c|c|}
\hline $\mathrm{C} 14-\mathrm{C} 19$ & $1.389(2)$ & $\mathrm{C} 18-\mathrm{C} 17$ & $1.367(3)$ \\
\hline $\mathrm{C} 1-\mathrm{C} 2$ & $1.370(3)$ & $\mathrm{C} 5-\mathrm{H} 5$ & 0.9300 \\
\hline $\mathrm{C} 1-\mathrm{C} 6$ & $1.374(3)$ & $\mathrm{C} 5-\mathrm{C} 6$ & $1.379(3)$ \\
\hline $\mathrm{C} 9-\mathrm{C} 8$ & $1.363(2)$ & C6-H6 & 0.9300 \\
\hline $\mathrm{C} 9-\mathrm{C} 11$ & $1.478(3)$ & C13-H13A & 0.9700 \\
\hline $\mathrm{C} 4-\mathrm{C} 3$ & $1.373(3)$ & C13-H13B & 0.9700 \\
\hline $\mathrm{C} 4-\mathrm{C} 5$ & $1.376(3)$ & $\mathrm{C} 13-\mathrm{C} 12$ & $1.457(3)$ \\
\hline $\mathrm{C} 10-\mathrm{C} 8$ & $1.476(3)$ & $\mathrm{C} 12-\mathrm{H} 12 \mathrm{~A}$ & 0.9700 \\
\hline $\mathrm{N} 3-\mathrm{C} 8$ & $1.357(2)$ & C12-H12B & 0.9700 \\
\hline $\mathrm{N} 3-\mathrm{N} 2$ & $1.303(2)$ & C7-H7A & 0.9600 \\
\hline $\mathrm{C} 15-\mathrm{H} 15$ & 0.9300 & C7-H7B & 0.9600 \\
\hline $\mathrm{C} 15-\mathrm{C} 16$ & $1.385(3)$ & $\mathrm{C} 7-\mathrm{H} 7 \mathrm{C}$ & 0.9600 \\
\hline $\mathrm{C} 4-\mathrm{O} 2-\mathrm{C} 7$ & $117.47(18)$ & $\mathrm{C} 15-\mathrm{C} 16-\mathrm{H} 16$ & 120.1 \\
\hline $\mathrm{C} 14-\mathrm{N} 4-\mathrm{H} 4$ & 115.9 & $\mathrm{C} 17-\mathrm{C} 16-\mathrm{C} 15$ & $119.8(2)$ \\
\hline $\mathrm{C} 10-\mathrm{N} 4-\mathrm{H} 4$ & 115.9 & $\mathrm{C} 17-\mathrm{C} 16-\mathrm{H} 16$ & 120.1 \\
\hline $\mathrm{C} 10-\mathrm{N} 4-\mathrm{C} 14$ & $128.10(18)$ & C9-C11-H11 & 113.1 \\
\hline $\mathrm{C} 9-\mathrm{N} 1-\mathrm{C} 1$ & 132.18 (19) & $\mathrm{C} 9-\mathrm{C} 11-\mathrm{C} 12$ & $124.1(2)$ \\
\hline $\mathrm{C} 9-\mathrm{N} 1-\mathrm{N} 2$ & $110.40(17)$ & $\mathrm{C} 13-\mathrm{C} 11-\mathrm{C} 9$ & $124.2(2)$ \\
\hline $\mathrm{N} 2-\mathrm{N} 1-\mathrm{C} 1$ & $117.40(17)$ & $\mathrm{C} 13-\mathrm{C} 11-\mathrm{H} 11$ & 113.1 \\
\hline $\mathrm{C} 15-\mathrm{C} 14-\mathrm{N} 4$ & $123.7(2)$ & $\mathrm{C} 13-\mathrm{C} 11-\mathrm{C} 12$ & 58.84 (14) \\
\hline $\mathrm{C} 15-\mathrm{C} 14-\mathrm{C} 19$ & $119.1(2)$ & $\mathrm{C} 12-\mathrm{C} 11-\mathrm{H} 11$ & 113.1 \\
\hline $\mathrm{C} 19-\mathrm{C} 14-\mathrm{N} 4$ & $117.2(2)$ & $\mathrm{C} 19-\mathrm{C} 18-\mathrm{H} 18$ & 120.2 \\
\hline $\mathrm{C} 2-\mathrm{C} 1-\mathrm{N} 1$ & $119.5(2)$ & $\mathrm{C} 17-\mathrm{C} 18-\mathrm{C} 19$ & $119.7(2)$ \\
\hline $\mathrm{C} 2-\mathrm{C} 1-\mathrm{C} 6$ & $120.7(2)$ & $\mathrm{C} 17-\mathrm{C} 18-\mathrm{H} 18$ & 120.2 \\
\hline $\mathrm{C} 6-\mathrm{C} 1-\mathrm{N} 1$ & $119.8(2)$ & $\mathrm{C} 16-\mathrm{C} 17-\mathrm{Cl1}$ & 119.63 (19) \\
\hline $\mathrm{N} 1-\mathrm{C} 9-\mathrm{C} 8$ & $103.98(17)$ & $\mathrm{C} 16-\mathrm{C} 17-\mathrm{C} 18$ & $120.8(2)$ \\
\hline $\mathrm{N} 1-\mathrm{C} 9-\mathrm{C} 11$ & $127.6(2)$ & $\mathrm{C} 18-\mathrm{C} 17-\mathrm{Cl1}$ & $119.56(18)$ \\
\hline $\mathrm{C} 8-\mathrm{C} 9-\mathrm{C} 11$ & $128.4(2)$ & $\mathrm{C} 4-\mathrm{C} 5-\mathrm{H} 5$ & 119.6 \\
\hline $\mathrm{O} 2-\mathrm{C} 4-\mathrm{C} 3$ & $124.7(2)$ & $\mathrm{C} 4-\mathrm{C} 5-\mathrm{C} 6$ & $120.8(2)$ \\
\hline $\mathrm{O} 2-\mathrm{C} 4-\mathrm{C} 5$ & $115.5(2)$ & $\mathrm{C} 6-\mathrm{C} 5-\mathrm{H} 5$ & 119.6 \\
\hline $\mathrm{C} 3-\mathrm{C} 4-\mathrm{C} 5$ & $119.9(2)$ & $\mathrm{C} 1-\mathrm{C} 6-\mathrm{C} 5$ & $119.1(2)$ \\
\hline $\mathrm{O} 1-\mathrm{C} 10-\mathrm{N} 4$ & 124.08 (19) & $\mathrm{C} 1-\mathrm{C} 6-\mathrm{H} 6$ & 120.5 \\
\hline $\mathrm{O} 1-\mathrm{C} 10-\mathrm{C} 8$ & $122.9(2)$ & $\mathrm{C} 5-\mathrm{C} 6-\mathrm{H} 6$ & 120.5 \\
\hline $\mathrm{N} 4-\mathrm{C} 10-\mathrm{C} 8$ & $113.0(2)$ & $\mathrm{C} 11-\mathrm{C} 13-\mathrm{H} 13 \mathrm{~A}$ & 117.7 \\
\hline $\mathrm{N} 2-\mathrm{N} 3-\mathrm{C} 8$ & $108.94(17)$ & $\mathrm{C} 11-\mathrm{C} 13-\mathrm{H} 13 \mathrm{~B}$ & 117.7 \\
\hline $\mathrm{C} 14-\mathrm{C} 15-\mathrm{H} 15$ & 119.9 & $\mathrm{H} 13 \mathrm{~A}-\mathrm{C} 13-\mathrm{H} 13 \mathrm{~B}$ & 114.8 \\
\hline $\mathrm{C} 14-\mathrm{C} 15-\mathrm{C} 16$ & $120.2(2)$ & $\mathrm{C} 12-\mathrm{C} 13-\mathrm{C} 11$ & $61.14(16)$ \\
\hline $\mathrm{C} 16-\mathrm{C} 15-\mathrm{H} 15$ & 119.9 & $\mathrm{C} 12-\mathrm{C} 13-\mathrm{H} 13 \mathrm{~A}$ & 117.7 \\
\hline $\mathrm{C} 9-\mathrm{C} 8-\mathrm{C} 10$ & $130.9(2)$ & $\mathrm{C} 12-\mathrm{C} 13-\mathrm{H} 13 \mathrm{~B}$ & 117.7 \\
\hline $\mathrm{N} 3-\mathrm{C} 8-\mathrm{C} 9$ & $109.70(18)$ & $\mathrm{C} 11-\mathrm{C} 12-\mathrm{H} 12 \mathrm{~A}$ & 117.8 \\
\hline $\mathrm{N} 3-\mathrm{C} 8-\mathrm{C} 10$ & $119.4(2)$ & $\mathrm{C} 11-\mathrm{C} 12-\mathrm{H} 12 \mathrm{~B}$ & 117.8 \\
\hline $\mathrm{C} 4-\mathrm{C} 3-\mathrm{H} 3$ & 120.2 & $\mathrm{C} 13-\mathrm{C} 12-\mathrm{C} 11$ & $60.02(15)$ \\
\hline $\mathrm{C} 4-\mathrm{C} 3-\mathrm{C} 2$ & $119.6(2)$ & $\mathrm{C} 13-\mathrm{C} 12-\mathrm{H} 12 \mathrm{~A}$ & 117.8 \\
\hline $\mathrm{C} 2-\mathrm{C} 3-\mathrm{H} 3$ & 120.2 & $\mathrm{C} 13-\mathrm{C} 12-\mathrm{H} 12 \mathrm{~B}$ & 117.8 \\
\hline $\mathrm{N} 3-\mathrm{N} 2-\mathrm{N} 1$ & $106.97(17)$ & $\mathrm{H} 12 \mathrm{~A}-\mathrm{C} 12-\mathrm{H} 12 \mathrm{~B}$ & 114.9 \\
\hline $\mathrm{C} 14-\mathrm{C} 19-\mathrm{H} 19$ & 119.8 & $\mathrm{O} 2-\mathrm{C} 7-\mathrm{H} 7 \mathrm{~A}$ & 109.5 \\
\hline $\mathrm{C} 18-\mathrm{C} 19-\mathrm{C} 14$ & $120.4(2)$ & $\mathrm{O} 2-\mathrm{C} 7-\mathrm{H} 7 \mathrm{~B}$ & 109.5 \\
\hline
\end{tabular}




\begin{tabular}{|c|c|c|c|}
\hline $\mathrm{C} 18-\mathrm{C} 19-\mathrm{H} 19$ & 119.8 & $\mathrm{O} 2-\mathrm{C} 7-\mathrm{H} 7 \mathrm{C}$ & 109.5 \\
\hline $\mathrm{C} 1-\mathrm{C} 2-\mathrm{C} 3$ & $120.0(2)$ & $\mathrm{H} 7 \mathrm{~A}-\mathrm{C} 7-\mathrm{H} 7 \mathrm{~B}$ & 109.5 \\
\hline $\mathrm{C} 1-\mathrm{C} 2-\mathrm{H} 2$ & 120.0 & $\mathrm{H} 7 \mathrm{~A}-\mathrm{C} 7-\mathrm{H} 7 \mathrm{C}$ & 109.5 \\
\hline $\mathrm{C} 3-\mathrm{C} 2-\mathrm{H} 2$ & 120.0 & $\mathrm{H} 7 \mathrm{~B}-\mathrm{C} 7-\mathrm{H} 7 \mathrm{C}$ & 109.5 \\
\hline $\mathrm{O} 1-\mathrm{C} 10-\mathrm{C} 8-\mathrm{C} 9$ & $6.7(4)$ & $\mathrm{C} 4-\mathrm{C} 5-\mathrm{C} 6-\mathrm{C} 1$ & $0.4(3)$ \\
\hline $\mathrm{O} 1-\mathrm{C} 10-\mathrm{C} 8-\mathrm{N} 3$ & $-173.6(2)$ & $\mathrm{C} 10-\mathrm{N} 4-\mathrm{C} 14-\mathrm{C} 15$ & $23.3(4)$ \\
\hline $\mathrm{O} 2-\mathrm{C} 4-\mathrm{C} 3-\mathrm{C} 2$ & $179.10(19)$ & $\mathrm{C} 10-\mathrm{N} 4-\mathrm{C} 14-\mathrm{C} 19$ & $-158.0(2)$ \\
\hline $\mathrm{O} 2-\mathrm{C} 4-\mathrm{C} 5-\mathrm{C} 6$ & $-179.5(2)$ & $\mathrm{C} 15-\mathrm{C} 14-\mathrm{C} 19-\mathrm{C} 18$ & $1.7(3)$ \\
\hline $\mathrm{N} 4-\mathrm{C} 14-\mathrm{C} 15-\mathrm{C} 16$ & $177.1(2)$ & $\mathrm{C} 15-\mathrm{C} 16-\mathrm{C} 17-\mathrm{Cl1}$ & $-178.77(17)$ \\
\hline N4 $-\mathrm{C} 14-\mathrm{C} 19-\mathrm{C} 18$ & $-177.10(19)$ & $\mathrm{C} 15-\mathrm{C} 16-\mathrm{C} 17-\mathrm{C} 18$ & $0.6(3)$ \\
\hline $\mathrm{N} 4-\mathrm{C} 10-\mathrm{C} 8-\mathrm{C} 9$ & $-171.8(2)$ & $\mathrm{C} 8-\mathrm{C} 9-\mathrm{C} 11-\mathrm{C} 13$ & $-139.8(3)$ \\
\hline $\mathrm{N} 4-\mathrm{C} 10-\mathrm{C} 8-\mathrm{N} 3$ & $7.9(3)$ & $\mathrm{C} 8-\mathrm{C} 9-\mathrm{C} 11-\mathrm{C} 12$ & $147.4(3)$ \\
\hline $\mathrm{N} 1-\mathrm{C} 1-\mathrm{C} 2-\mathrm{C} 3$ & $177.23(19)$ & $\mathrm{C} 8-\mathrm{N} 3-\mathrm{N} 2-\mathrm{N} 1$ & $-0.5(3)$ \\
\hline $\mathrm{N} 1-\mathrm{C} 1-\mathrm{C} 6-\mathrm{C} 5$ & $-177.6(2)$ & $\mathrm{C} 3-\mathrm{C} 4-\mathrm{C} 5-\mathrm{C} 6$ & $0.8(3)$ \\
\hline $\mathrm{N} 1-\mathrm{C} 9-\mathrm{C} 8-\mathrm{C} 10$ & $179.6(2)$ & $\mathrm{N} 2-\mathrm{N} 1-\mathrm{C} 1-\mathrm{C} 2$ & $-86.6(2)$ \\
\hline $\mathrm{N} 1-\mathrm{C} 9-\mathrm{C} 8-\mathrm{N} 3$ & $-0.1(3)$ & $\mathrm{N} 2-\mathrm{N} 1-\mathrm{C} 1-\mathrm{C} 6$ & $89.8(3)$ \\
\hline $\mathrm{N} 1-\mathrm{C} 9-\mathrm{C} 11-\mathrm{C} 13$ & $41.2(4)$ & $\mathrm{N} 2-\mathrm{N} 1-\mathrm{C} 9-\mathrm{C} 8$ & $-0.2(2)$ \\
\hline $\mathrm{N} 1-\mathrm{C} 9-\mathrm{C} 11-\mathrm{C} 12$ & $-31.6(4)$ & $\mathrm{N} 2-\mathrm{N} 1-\mathrm{C} 9-\mathrm{C} 11$ & $179.1(2)$ \\
\hline $\mathrm{C} 14-\mathrm{N} 4-\mathrm{C} 10-\mathrm{O} 1$ & $1.2(4)$ & $\mathrm{N} 2-\mathrm{N} 3-\mathrm{C} 8-\mathrm{C} 9$ & $0.4(3)$ \\
\hline $\mathrm{C} 14-\mathrm{N} 4-\mathrm{C} 10-\mathrm{C} 8$ & $179.7(2)$ & $\mathrm{N} 2-\mathrm{N} 3-\mathrm{C} 8-\mathrm{C} 10$ & $-179.4(2)$ \\
\hline $\mathrm{C} 14-\mathrm{C} 15-\mathrm{C} 16-\mathrm{C} 17$ & $0.5(3)$ & $\mathrm{C} 19-\mathrm{C} 14-\mathrm{C} 15-\mathrm{C} 16$ & $-1.6(3)$ \\
\hline $\mathrm{C} 14-\mathrm{C} 19-\mathrm{C} 18-\mathrm{C} 17$ & $-0.7(3)$ & $\mathrm{C} 19-\mathrm{C} 18-\mathrm{C} 17-\mathrm{Cl1}$ & $178.88(17)$ \\
\hline $\mathrm{C} 1-\mathrm{N} 1-\mathrm{C} 9-\mathrm{C} 8$ & $178.1(2)$ & $\mathrm{C} 19-\mathrm{C} 18-\mathrm{C} 17-\mathrm{C} 16$ & $-0.5(3)$ \\
\hline $\mathrm{C} 1-\mathrm{N} 1-\mathrm{C} 9-\mathrm{C} 11$ & $-2.7(4)$ & $\mathrm{C} 2-\mathrm{C} 1-\mathrm{C} 6-\mathrm{C} 5$ & $-1.2(3)$ \\
\hline $\mathrm{C} 1-\mathrm{N} 1-\mathrm{N} 2-\mathrm{N} 3$ & $-178.2(2)$ & $\mathrm{C} 11-\mathrm{C} 9-\mathrm{C} 8-\mathrm{C} 10$ & $0.4(4)$ \\
\hline $\mathrm{C} 9-\mathrm{N} 1-\mathrm{C} 1-\mathrm{C} 2$ & $95.2(3)$ & $\mathrm{C} 11-\mathrm{C} 9-\mathrm{C} 8-\mathrm{N} 3$ & $-179.3(2)$ \\
\hline $\mathrm{C} 9-\mathrm{N} 1-\mathrm{C} 1-\mathrm{C} 6$ & $-88.4(3)$ & $\mathrm{C} 5-\mathrm{C} 4-\mathrm{C} 3-\mathrm{C} 2$ & $-1.2(3)$ \\
\hline $\mathrm{C} 9-\mathrm{N} 1-\mathrm{N} 2-\mathrm{N} 3$ & $0.4(3)$ & $\mathrm{C} 6-\mathrm{C} 1-\mathrm{C} 2-\mathrm{C} 3$ & $0.8(3)$ \\
\hline $\mathrm{C} 9-\mathrm{C} 11-\mathrm{C} 13-\mathrm{C} 12$ & $-112.4(3)$ & $\mathrm{C} 7-\mathrm{O} 2-\mathrm{C} 4-\mathrm{C} 3$ & $8.1(3)$ \\
\hline $\mathrm{C} 9-\mathrm{C} 11-\mathrm{C} 12-\mathrm{C} 13$ & $112.6(3)$ & $\mathrm{C} 7-\mathrm{O} 2-\mathrm{C} 4-\mathrm{C} 5$ & $-171.63(19)$ \\
\hline $\mathrm{C} 4-\mathrm{C} 3-\mathrm{C} 2-\mathrm{C} 1$ & $0.4(3)$ & & \\
\hline
\end{tabular}

Hydrogen-bond geometry $\left(A,{ }^{\circ}\right)$

\begin{tabular}{lllll}
\hline$D-\mathrm{H} \cdots A$ & $D-\mathrm{H}$ & $\mathrm{H} \cdots A$ & $D \cdots A$ & $D-\mathrm{H} \cdots A$ \\
\hline $\mathrm{N} 4-\mathrm{H} 4 \cdots \mathrm{N} 3$ & 0.86 & 2.24 & $2.680(3)$ & 112 \\
$\mathrm{~N} 4-\mathrm{H} 4 \cdots \mathrm{N} 2^{\mathrm{i}}$ & 0.86 & 2.68 & $3.491(2)$ & 157 \\
$\mathrm{C} 15-\mathrm{H} 15 \cdots \mathrm{O} 1$ & 0.93 & 2.39 & $2.936(2)$ & 117 \\
$\mathrm{C} 19-\mathrm{H} 19 \cdots \mathrm{N} 2^{\mathrm{i}}$ & 0.93 & 2.68 & $3.475(3)$ & 144 \\
$\mathrm{C} 2-\mathrm{H} 2 \cdots \mathrm{O} 1^{\mathrm{ii}}$ & 0.93 & 2.53 & $3.439(3)$ & 167 \\
$\mathrm{C} 11-\mathrm{H} 11 \cdots \mathrm{O} 1$ & 0.98 & 2.47 & $3.124(2)$ & 124 \\
\hline
\end{tabular}

Symmetry codes: (i) $-x+1,-y,-z+1$; (ii) $-x+1,-y+1,-z+1$. 